DSF-T-92/23

INFN-NA-IV-92/23

\title{
Nonleptonic Cabibbo favoured B-decays and CP-asymmetries for Charmed final hadron states in Isgur and Wise theory
}

\author{
F. Buccella ${ }^{1}$, F. Lombardi ${ }^{1}$, G. Miele ${ }^{1,2}$ and P. Santorelli ${ }^{1,2}$ \\ ${ }^{1}$ Dipartimento di Scienze Fisiche ,Università di Napoli, Mostra D’Oltremare pad.19, I-80125 Napoli, Italy \\ ${ }^{2}$ INFN, Sezione di Napoli, Mostra D’Oltremare pad.20, I-80125 Napoli, Italy
}

PACS: 14.40.J; 13.25

\begin{abstract}
The Cabibbo allowed non-leptonic $B$-decays in two hadrons are studied, within the factorization hypothesis, in the framework of Isgur and Wise theory for the matrix elements of the $\Delta B=-\Delta C= \pm 1$ weak currents. The $S U(2)_{H F}$ symmetry relates $|\Delta B|=1$ to $|\Delta C|=1$ currents, which have been measured in the semileptonic strange decays of charmed particles. By assuming colour screening and allowing for $S U(3)$ invariant contributions from the annihilation terms with charmed final states one is able to comply with the present experimental knowledge.

The $C P$ violating asymmetries in neutral $B$ decays are given for charmed final states in terms of the $K-M$ angles. With the central values found for the annihilation parameters there is a destructive (constructive) interference between the direct and annihilation terms in the Cabibbo allowed (doubly forbidden) amplitudes for the decays into $D^{0}\left(D^{* 0}\right) \pi^{0}$ and $D^{0} \rho^{0}$ so that they may be of the same order. This would imply large asymmetries, for which however our present knowledge on the amplitudes does not allow to predict even their sign.

We have better confidence in our predictions for the charged final states than the neutral ones and can draw the conclusion that the detection of the corresponding asymmetries requires, at least, $10^{6}$ tagged neutral $B$-particles.
\end{abstract}

\footnotetext{
${ }^{1}$ To be published in Zeitschrift für Physik $C$.
} 


\section{Introduction}

The study of the semileptonic decays of $B$ particles in final hadrons with $|C|=1,0$ provides a laboratory to test Isgur and Wise theory [1]. Following Neubert [2] we can get for $\left|V_{b c}\right|$, by an extrapolation of the data [3] on the semileptonic decay $\bar{B}_{d}^{0} \rightarrow D^{+*} e^{-} \bar{\nu}_{e}$, the range of values $0.044 \div 0.052$ in agreement with previous determinations.

The smallness of the element $V_{b u}$ does not allow, at the moment, to have information about $\Delta B=1$ and $\Delta C=0$ matrix elements of the vector and axial currents. A larger domain to compare theory with the experiment would be obtained in the framework of the factorization hypothesis since the matrix elements of the $\Delta B=1$ weak currents appear in the amplitudes of the exclusive two-body nonleptonic decays. In a recent paper [4] about nonleptonic decays of charmed particles one has been able to find a reasonable fit to the amplitudes for $P P, P V$ and $P A$ final states by keeping into account the final state interaction and by taking as a free parameters the matrix elements of the divergences of the vector and axial currents between the vacuum and two hadrons final states, which appear in the annihilation terms. For the amplitudes of $B$ decays we neglect the final state interaction of the two hadrons, due to the high energy of the decay products, and, as for charmed decays, we take as free parameters, related by $S U(3)$ symmetry, the matrix elements of the divergences of the weak currents between the vacuum and the two mesons $|C|=1$ final states.

The weak amplitudes, within the factorization approximation, depend on the matrix elements of the weak currents between $B=1$ and $C=1$ mesons, which may be written in terms of the universal Isgur-Wise function $\xi\left(w^{2}\right)[1]$, or mesons built with light quarks $(u, d$ and $s)$.

The E691 Collaboration [5] has studied the lepton spectrum in the semileptonic decays of charmed particles in $S=1$ final hadron state and found the residua of the relevant form factors within the hypothesis of pole dominance and with the position of the pole dictated by the lowest resonance with the right quantum numbers. The matrix elements between $B=1$ and the light mesons may be related to the ones, given in [5], according to $S U(2)_{H F}[6],[7]$. In conclusion we are able to predict the amplitudes for Cabibbo favoured decays of $B$ mesons, for which one has some experimental knowledge, in terms of the Isgur-Wise function $\xi\left(w^{2}\right)$ of the three parameters appearing in the annihilation terms and in terms of the $|\Delta C|=1$ form factors, which have been measured by E691 Collaboration. We shall take the parabolic expression previously proposed [8] for $\xi\left(w^{2}\right)=1+b w^{2}+c w^{4}$ as well as different choices [9] to show the dependence of the results on the parameterization.

In previous works [10] we studied the $C P$ violating asymmetries expected in the decays of the neutral $B$ particles still in the framework of the factorization approximation and neglecting the final state interaction, but with different assumptions on the matrix elements of the weak currents. Within the theoretical framework described here we are able to predict the asymmetries for $|C|=1$ final states and compare them with our previous papers. 
The paper is organized as follows. In section 1 we give the effective hamiltonian for the Cabibbo favoured nonleptonic decays of $|B|=1$ particles, the relevant matrix elements of the $\Delta B=-\Delta C= \pm 1$ form factors vector and axial current in the Isgur and Wise theory, in terms of $\xi\left(w^{2}\right)$ and the relationships between $\Delta B= \pm 1$ and $\Delta C= \pm 1$ form factors deduced from $S U(2)_{H F}$ in the framework Heavy Quark Effective Theory (HQET) [6],[11].

In the second section we compute some of the considered amplitudes and we define the free parameters appearing in the annihilation terms. For $\xi\left(w^{2}\right)$ we assume the parabolic, exponential and pole form respectively (with $\xi(0)=1$ ), whereas the form factors, which appear in $\Delta B=1$ currents, will be written in terms of the experimentally fitted for $\Delta C=1$. Furthermore, releasing the $\xi(0)=1$ constraint (dictated by $H Q E T$ ) we shall also study the agreement with the experimental data of the theoretical predictions obtained assuming for $\xi\left(w^{2}\right)$ a physical pole expression.

In the third section we shall give our predictions for the $C P$ violating asymmetries for $C= \pm 1$ final states and show their dependence on the parameters appearing in the annihilation terms.

Finally we give our conclusions.

\section{Matrix elements of $\Delta B= \pm 1$ weak currents in HQET}

The bare weak Hamiltonian for the $|\Delta B|=1$ Cabibbo favoured nonleptonic decays is:

$$
H^{\Delta B= \pm 1}=-i \frac{4 G_{F}}{\sqrt{2}} V_{b c}\left(\bar{c}_{L} \gamma_{\mu} b_{L}\right)\left(V_{s c}^{*} \bar{s}_{L} \gamma^{\mu} c_{L}+V_{d u}^{*} \bar{d}_{L} \gamma^{\mu} u_{L}\right)+\text { h.c. }
$$

The short-range $Q C D$ corrections at subleading -log approximation have been computed by the rinormalization group approach [12].

We can get $H_{e f f}^{\Delta B= \pm 1}$ from (1) by performing the following substitution ?

$$
\begin{aligned}
\bar{c}_{L} \gamma_{\mu} b_{L} \bar{q}_{2 L} \gamma^{\mu} q_{1 L} & \rightarrow k_{1} \bar{c}_{L} \gamma_{\mu} b_{L} \bar{q}_{2 L} \gamma^{\mu} q_{1 L} \\
& +k_{2} \bar{q}_{2 L} \gamma_{\mu} b_{L} \bar{c}_{L} \gamma^{\mu} q_{1 L}
\end{aligned}
$$

with $q_{1} \equiv c($ or $u), q_{2} \equiv s($ or $d)$ and $k_{1}, k_{2}$ given by:

$$
\begin{aligned}
C_{+} & =\left(\frac{\alpha_{s}\left(m_{b}\right)}{\alpha_{s}\left(m_{W}\right)}\right)^{-\frac{6}{23}}\left(1-0.51 \frac{\alpha_{s}\left(m_{b}\right)-\alpha_{s}\left(m_{W}\right)}{\pi}\right) \\
C_{-} & =\left(\frac{\alpha_{s}\left(m_{b}\right)}{\alpha_{s}\left(m_{W}\right)}\right)^{\frac{12}{23}}\left(1+1.48 \frac{\alpha_{s}\left(m_{b}\right)-\alpha_{s}\left(m_{W}\right)}{\pi}\right) \\
k_{1} & \equiv \frac{1}{2}\left(C_{+}+C_{-}\right) \cong 1.11 \\
k_{2} & \equiv \frac{1}{2}\left(C_{+}-C_{-}\right) \cong-0.25
\end{aligned}
$$

\footnotetext{
${ }^{2}$ We neglect the penguin contribution to the $\Delta B=\Delta S= \pm 1$ amplitudes since the small coefficient $\approx 0.028$ for the corresponding operator is not compensated, as in the decays of strange particles, by an enhancement for the matrix elements, since the final hadrons do not contain quarks of the first family.
} 
The values for $\alpha_{s}\left(m_{b}\right)$ and $\alpha_{s}\left(M_{W}\right)$ are taken consistently with the value for $\alpha_{s}\left(m_{c}\right)$ of a previous work on the nonleptonic decays of $D$ particles [4]:

$$
\alpha_{s}\left(m_{c}\right)=0.272 \quad \alpha_{s}\left(m_{b}\right)=0.187 \quad \alpha_{s}\left(M_{W}\right)=0.110
$$

In the factorization approximation the matrix elements of $H_{e f f}^{\Delta B= \pm 1}$ are given in terms of the matrix elements of the vector and axial currents as:

$$
\begin{aligned}
& \left\langle D^{0}\left|\bar{c} \gamma_{\mu} b\right| B^{-}\right\rangle \quad\left\langle D^{* 0}\left|\bar{c} \gamma_{\mu} \gamma_{5} b\right| B^{-}\right\rangle \\
& \left\langle K^{-}\left|\bar{s} \gamma_{\mu} b\right| B^{-}\right\rangle \quad\left\langle\rho^{-}\left|\bar{d} \gamma_{\mu} \gamma_{5} b\right| B^{-}\right\rangle \\
& \left\langle D^{-} \bar{K}^{0}\left|\partial^{\mu} \bar{s} \gamma_{\mu} c\right| 0\right\rangle \quad\left\langle D^{*-} \bar{K}^{0}\left|\partial^{\mu} \bar{s} \gamma_{\mu} \gamma_{5} c\right| 0\right\rangle \\
& \left\langle 0\left|\bar{d} \gamma_{\mu} \gamma_{5} b\right| \bar{B}_{d}^{0}\right\rangle
\end{aligned}
$$

The matrix elements between charmed and beautiful mesons are given in terms of the universal form factor $\xi\left(w^{2}\right)$ according to Isgur and Wise theory [11]:

$$
\begin{aligned}
& \left\langle D\left(v^{\prime}\right)\left|\bar{c} \gamma_{\mu} b\right| B(v)\right\rangle=C_{b c} \sqrt{m_{B} m_{D}} \xi\left(w^{2}\right)\left(v_{\mu}+v_{\mu}^{\prime}\right) \\
& \left\langle D^{*}\left(v^{\prime}, \varepsilon\right)\left|\bar{c} \gamma_{\mu} \gamma_{5} b\right| B(v)\right\rangle=i C_{b c} \sqrt{m_{B} m_{D^{*}}} \xi\left(w^{2}\right)\left[\varepsilon_{\mu}^{*}\left(1+v \cdot v^{\prime}\right)-\left(\varepsilon^{*} \cdot v\right) v_{\mu}^{\prime}\right]
\end{aligned}
$$

where $v$ and $v^{\prime}$ are the quadri-velocities of the heavy hadrons and $C_{b c}$, neglecting the dependence on $v \cdot v^{\prime}$, is given by [1],[11]:

$$
C_{b c}=\left(\frac{\alpha_{s}\left(m_{b}\right)}{\alpha_{s}\left(m_{c}\right)}\right)^{-6 / 25} \cong 1.10
$$

The matrix elements between the $B=1$ and the light mesons $q \in\{u, d, s\}$ may be written in the general form:

$$
\begin{aligned}
\left\langle P_{i}\left(p_{i}\right)\left|\bar{q} \gamma^{\mu} b\right| B(v)\right\rangle & =f_{+}^{(b q)}\left(q^{2}\right)\left(m_{B} v^{\mu}+p_{i}^{\mu}\right)+f_{-}^{(b q)}\left(q^{2}\right)\left(m_{B} v^{\mu}-p_{i}^{\mu}\right) \\
\left\langle V_{j}\left(p_{j}, \varepsilon\right)\left|\bar{q} \gamma^{\mu} \gamma_{5} b\right| B(v)\right\rangle & =i f^{(b q)}\left(q^{2}\right) \varepsilon^{* \mu}+i a_{+}^{(b q)}\left(q^{2}\right) \varepsilon^{*} \cdot v m_{B}\left(m_{B} v^{\mu}+p_{j}^{\mu}\right) \\
& +i a_{-}^{(b q)}\left(q^{2}\right) \varepsilon^{*} \cdot v m_{B}\left(m_{B} v^{\mu}-p_{j}^{\mu}\right)
\end{aligned}
$$

and in the same way for $C=1$ :

$$
\begin{aligned}
\left\langle P_{i}\left(p_{i}\right)\left|\bar{q} \gamma^{\mu} c\right| D(v)\right\rangle & =f_{+}^{(c q)}\left(q^{2}\right)\left(m_{D} v^{\mu}+p_{i}^{\mu}\right)+f_{-}^{(c q)}\left(q^{2}\right)\left(m_{D} v^{\mu}-p_{i}^{\mu}\right) \\
\left\langle V_{j}\left(p_{j}, \varepsilon\right)\left|\bar{q} \gamma^{\mu} \gamma_{5} c\right| D(v)\right\rangle & =i f^{(c q)}\left(q^{2}\right) \varepsilon^{* \mu}+i a_{+}^{(c q)}\left(q^{2}\right) \varepsilon^{*} \cdot v m_{D}\left(m_{D} v^{\mu}+p_{j}^{\mu}\right) \\
& +i a_{-}^{(c q)}\left(q^{2}\right) \varepsilon^{*} \cdot v m_{D}\left(m_{D} v^{\mu}-p_{j}^{\mu}\right) .
\end{aligned}
$$

The symmetry $S U(2)_{H F}$ allows to relate the form factors introduced in (9) and (10] [6]:

$$
f_{+}^{(b q)}\left(q_{B}^{2}\right)=\frac{C_{b c}}{2 \sqrt{m_{B} m_{D}}}\left[\left(m_{B}+m_{D}\right) f_{+}^{(c q)}\left(q_{D}^{2}\right)+\left(m_{D}-m_{B}\right) f_{-}^{(c q)}\left(q_{D}^{2}\right)\right]
$$




$$
\begin{aligned}
& f_{-}^{(b q)}\left(q_{B}^{2}\right)=\frac{C_{b c}}{2 \sqrt{m_{B} m_{D}}}\left[\left(m_{D}-m_{B}\right) f_{+}^{(c q)}\left(q_{D}^{2}\right)+\left(m_{B}+m_{D}\right) f_{-}^{(c q)}\left(q_{D}^{2}\right)\right] \\
& a_{+}^{(b q)}\left(q_{B}^{2}\right)=\frac{C_{b c}}{2} \sqrt{\frac{m_{D}}{m_{B}}}\left[\left(\frac{m_{D}}{m_{B}}+1\right) a_{+}^{(c q)}\left(q_{D}^{2}\right)+\left(\frac{m_{D}}{m_{B}}-1\right) a_{-}^{(c q)}\left(q_{D}^{2}\right)\right] \\
& a_{-}^{(b q)}\left(q_{B}^{2}\right)=\frac{C_{b c}}{2} \sqrt{\frac{m_{D}}{m_{B}}}\left[\left(\frac{m_{D}}{m_{B}}-1\right) a_{+}^{(c q)}\left(q_{D}^{2}\right)+\left(\frac{m_{D}}{m_{B}}+1\right) a_{-}^{(c q)}\left(q_{D}^{2}\right)\right] \\
& f^{(b q)}\left(q_{B}^{2}\right)=C_{b c} \sqrt{\frac{m_{B}}{m_{D}}} f^{(c q)}\left(q_{D}^{2}\right)
\end{aligned}
$$

where $C_{b c}$ is given by (8) and

$$
\begin{aligned}
q_{B}^{2} & =\left(m_{B} v-p_{i(j)}\right)^{2} \\
q_{D}^{2} & =\left(m_{D} v-p_{i(j)}\right)^{2}
\end{aligned}
$$

The form factors for charmed and beautiful particles in (9) and (10) are related to the ones defined in the helicity frame of reference (see for example [13]) by:

$$
\begin{aligned}
& f_{+}^{(Q q)}\left(q^{2}\right)=f_{1}^{(Q q)}\left(q^{2}\right) \\
& f_{-}^{(Q q)}\left(q^{2}\right)=\frac{m_{1}^{2}-m_{2}^{2}}{q^{2}}\left[f_{0}^{(Q q)}\left(q^{2}\right)-f_{1}^{(Q q)}\left(q^{2}\right)\right] \\
& f^{(Q q)}\left(q^{2}\right)=\left(m_{1}+m_{2}\right) A_{1}^{(Q q)}\left(q^{2}\right) \\
& a_{+}^{(Q q)}\left(q^{2}\right)=-\frac{A_{2}^{(Q q)}\left(q^{2}\right)}{m_{1}+m_{2}} \\
& a_{-}^{(Q q)}\left(q^{2}\right)=-\frac{1}{q^{2}}\left[\left(m_{1}+m_{2}\right) A_{1}^{(Q q)}\left(q^{2}\right)-\left(m_{1}-m_{2}\right) A_{2}^{(Q q)}\left(q^{2}\right)+2 m_{2} A_{0}^{(Q q)}\left(q^{2}\right)\right]
\end{aligned}
$$

where $m_{1}$ and $m_{2}$ stand for the masses of the initial and final particle respectively; whereas $Q \in\{b, c\}$ and $q \in\{u, d, s\}$.

The experimental data for the semileptonic decays of $D$ particles with $S=-1$ in the final state, allow to fit the form factors defined in the r.h.s.'s of (13) in the case of $Q=c$ and $q=s$, by assuming for them a pole behaviour dominated by the lower resonance with the proper quantum numbers and leaving the residua as free parameters [5]. We extend these results to $q=d(u)$, applying $S U(3)$ symmetry to the above residua and using the correct masses for the involved resonances.

The results found are reported in Table 1.

\section{Evaluation of the amplitudes and comparison with experiment}

We write the amplitudes for the nonleptonic decays of $B$ particles for some particular processes assuming 
a complete colour screening 防:

$$
\begin{aligned}
& \mathcal{A}\left(B^{-} \rightarrow D^{0} \pi^{-}\right)=i \frac{G_{F}}{\sqrt{2}} V_{b c} V_{d u}^{*}\left[k_{1}\left\langle D^{0}\left|\bar{c} \gamma_{\mu} b\right| B^{-}\right\rangle\left\langle\pi^{-}\left|\bar{d} \gamma^{\mu} \gamma_{5} u\right| 0\right\rangle\right. \\
& \left.+\quad k_{2}\left\langle\pi^{-}\left|\bar{d} \gamma_{\mu} b\right| B^{-}\right\rangle\left\langle D^{0}\left|\bar{c} \gamma_{\mu} \gamma_{5} u\right| 0\right\rangle\right] \\
& =\frac{G_{F}}{\sqrt{2}} V_{b c} V_{d u}^{*}\left[k_{1} C_{b c} \sqrt{m_{B} m_{D}} \xi\left(w_{\pi}^{2}\right) f_{\pi}\left(m_{B}-m_{D}\right)\left(1+\frac{E_{D}}{m_{D}}\right)\right. \\
& \left.+k_{2} f_{0}^{(b d)}\left(m_{D}^{2}\right) f_{D}\left(m_{B}^{2}-m_{\pi}^{2}\right)\right] \\
& \mathcal{A}\left(\bar{B}_{d}^{0} \rightarrow D^{+} \pi^{-}\right)=i \frac{G_{F}}{\sqrt{2}} V_{b c} V_{d u}^{*}\left[k_{1}\left\langle D^{+}\left|\bar{c} \gamma_{\mu} b\right| \bar{B}_{d}^{0}\right\rangle\left\langle\pi^{-}\left|\bar{d} \gamma^{\mu} \gamma_{5} u\right| 0\right\rangle\right. \\
& \left.+\quad k_{2}\left\langle D^{+} \pi^{-}\left|\bar{c} \gamma_{\mu} u\right| 0\right\rangle\left\langle 0\left|\bar{d} \gamma^{\mu} \gamma_{5} b\right| \bar{B}_{d}^{0}\right\rangle\right] \\
& =\frac{G_{F}}{\sqrt{2}} V_{b c} V_{d u}^{*}\left[k_{1} C_{b c} \sqrt{m_{B} m_{D}} \xi\left(w_{\pi}^{2}\right) f_{\pi}\left(m_{B}-m_{D}\right)\left(1+\frac{E_{D}}{m_{D}}\right)\right. \\
& \left.-\quad k_{2}\left(m_{c}-m_{u}\right) W_{P P} m_{B}^{2}\right] \\
& \mathcal{A}\left(\bar{B}_{d}^{0} \rightarrow D^{0} \rho^{0}\right)=-i \frac{G_{F}}{\sqrt{2}} V_{b c} V_{d u}^{*} k_{2}\left[\left\langle\rho^{0}\left|\bar{d} \gamma_{\mu} \gamma_{5} b\right| \bar{B}_{d}^{0}\right\rangle\left\langle D^{0}\left|\bar{c} \gamma^{\mu} \gamma_{5} u\right| 0\right\rangle\right. \\
& \left.+\left\langle D^{0} \rho^{0}\left|\bar{c} \gamma_{\mu} \gamma_{5} u\right| 0\right\rangle\left\langle 0\left|\bar{d} \gamma^{\mu} \gamma_{5} b\right| \bar{B}_{d}^{0}\right\rangle\right] \\
& =-i \frac{G_{F}}{\sqrt{2}} V_{b c} V_{d u}^{*} k_{2}\left(-\frac{1}{\sqrt{2}}\right)\left[A_{0}^{(b d)}\left(m_{D}^{2}\right) f_{D}\right. \\
& \left.-\left(m_{c}+m_{u}\right) W_{D V}\right] 2 m_{B} m_{\rho} \varepsilon \cdot v \\
& \mathcal{A}\left(\bar{B}_{d}^{0} \rightarrow D^{+*} \pi^{-}\right)=-i \frac{G_{F}}{\sqrt{2}} V_{b c} V_{d u}^{*}\left[k_{1}\left\langle D^{+*}\left|\bar{c} \gamma_{\mu} \gamma_{5} b\right| \bar{B}_{d}^{0}\right\rangle\left\langle\pi^{-}\left|\bar{d} \gamma^{\mu} \gamma_{5} u\right| 0\right\rangle\right. \\
& \left.+\quad k_{2}\left\langle D^{+*} \pi^{-}\left|\bar{c} \gamma_{\mu} \gamma_{5} u\right| 0\right\rangle\left\langle 0\left|\bar{d} \gamma^{\mu} \gamma_{5} b\right| \bar{B}_{d}^{0}\right\rangle\right] \\
& =-i \frac{G_{F}}{\sqrt{2}} V_{b c} V_{d u}^{*}\left[k_{1} C_{b c}\left(\frac{m_{B}+m_{D^{*}}}{2 \sqrt{m_{B} m_{D^{*}}}}\right) \xi\left(w_{\pi}^{2}\right) f_{\pi}\right. \\
& \left.-\quad k_{2}\left(m_{c}+m_{u}\right) W_{D^{*} P} \quad\right] 2 m_{B} m_{D^{*}} \cdot v
\end{aligned}
$$

In (14)-(17) we denote with $W_{P P}, W_{D V}$ and $W_{D^{*} P}$ the reduced matrix elements for the annihilation terms defined analogously as in [4]. By applying $S U(3)$ symmetry to the scalar and pseudoscalar densities one gets the corresponding contributions for the strange final states as $D_{s}^{+} K^{-}$. Note that the $S U(4)$ flavour symmetry would imply $W_{D V}=W_{D^{*} P}$.

From the experimental data on the exclusive branching ratios of the nonleptonic decays of $B$ particle [15], the rates of the semileptonic channel $\overline{B_{d}^{0}} \rightarrow D^{+} e^{-} \bar{\nu}_{e}[15]$, the spectrum of $\bar{B}^{0} \rightarrow D^{+*} e^{-} \bar{\nu}_{e}[3]$ and the ratio $\Gamma\left(B^{-} \rightarrow D_{L}^{0 *} e^{-} \bar{\nu}_{e}\right) / \Gamma\left(B^{-} \rightarrow D_{T}^{0 *} e^{-} \bar{\nu}_{e}\right)[16]$ we obtain the values for the free parameters of the chosen

\footnotetext{
${ }^{3}$ We extend to the $B$ particles nonleptonic decays, the results of $D$ mesons decay [4], which suggest a complete screening of the factorizable part of the amplitude proportional to $1 / N_{c}$ due to the non-factorizable one [14].

${ }^{4}$ The decay constants used are: $f_{D^{*}}=f_{D_{s}}=f_{D}=210 \mathrm{MeV}$ (Mannel et al. in [9]), $f_{J / \Psi}=382 \mathrm{MeV}[14], f_{\rho}=221 \mathrm{MeV}$, $f_{K^{*}}=f_{K}=170 \mathrm{MeV}$ and $f_{\pi}=132 \mathrm{MeV}$.
} 
expressions for $\xi\left(w^{2}\right)$ :

$$
\begin{aligned}
\xi^{\text {par }}\left(w^{2}\right) & \equiv 1+b w^{2}+c w^{4} \\
\xi^{\exp }\left(w^{2}\right) & \equiv \exp \left\{\beta w^{2}\right\} \\
\xi^{\text {pole }}\left(w^{2}\right) & \equiv\left(1-\frac{w^{2}}{w_{0}^{2}}\right)^{-1} \\
\xi_{\text {pole }}^{\text {phys. }}\left(w^{2}\right) & \equiv \xi(0)\left(1-\frac{w^{2}}{1.69^{2}}\right)^{-1}
\end{aligned}
$$

The values for the annihilation parameters $W_{P P}, W_{D V}$ and $W_{D^{*} P}$ are obtained from the experimental values of the rates $\bar{B}_{d}^{0} \rightarrow D^{+} \pi^{-}, \bar{B}_{d}^{0} \rightarrow D^{+} \rho^{-}$and $\bar{B}_{d}^{0} \rightarrow D^{+*} \pi^{-}$respectively and are consistent with the experimental upper limits on $\bar{B}_{d}^{0} \rightarrow D_{s}^{+} K^{-}$and $\bar{B}_{d}^{0} \rightarrow D^{0} \rho^{0}$. These results are shown in Table 2, where also we report the predictions for the $W$ 's following Fakirov and Stech approach [17],[10].

In Table 3, in correspondence to the values in Table 2, we compare the theoretical predictions and the experimental values for the nonleptonic widths of $B$ mesons decays. We put in the same box the rates which should be equal according to the $\Delta I=0$ selection rule, obeyed by the Cabibbo favoured part of the $\Delta B=\Delta S=1$ hamiltonian. The amplitudes for the decay channels $B^{-}, \bar{B}_{d}^{0} \rightarrow D \rho$ are constrained by the $\Delta I=1, \Delta I_{3}=-1$ selection rule to obey:

$$
\mathcal{A}\left(\bar{B}_{d}^{0} \rightarrow D^{+} \rho^{-}\right)-\mathcal{A}\left(B^{-} \rightarrow D^{0} \rho^{-}\right)=\sqrt{2} \mathcal{A}\left(\bar{B}_{d}^{0} \rightarrow D^{0} \rho^{0}\right)
$$

which is consistent with the data.

From Tables 2 and 3, one sees that the parabolic form (18) is slightly favoured with respect to the others. In the case of the physical pole expression for $\xi\left(w^{2}\right)(21)$ we find $\xi(0)=0.84 \pm 0.05$ three standard deviations away from the value 1 predicted by $C V C$ and $C A C$ symmetries of $H Q E T$, for which one expects breaking effects only of the order $\Lambda_{Q C D}^{2} / m_{c}^{2}$ and $\alpha_{s}\left(m_{c}\right) / \pi$. The central values for the $W$ 's come larger in magnitude than the predictions in [10], as it has been the case for charm decays, but in general they are consistent with a vanishing value with the only exception of $W_{P P}$ found in correspondence to the parabolic parameterization

for $\xi\left(w^{2}\right)$. The amplitude for $B \rightarrow J / \Psi K$, which does not depend on $\xi\left(w^{2}\right)$, is proportional to $f_{+}^{(b q)}\left(m_{J / \Psi}^{2}\right)$, which by $S U(2)_{H F}$ is given by (11) and (13) in terms of the $|\Delta C|=1$ form factors studied by E691.

The agreement of the predictions with the measured rates represents a positive test for $S U(2)_{H F}$.

\section{CP violation in $\Delta \mathrm{C}=1$ neutral beauty decays}

In previous papers [10] we studied the $C P$ violating asymmetries in the decays of neutral beautiful particles in a different theoretical framework, consisting in a pole behaviour for all the $\Delta B=1$ form factors, $S U$ (4) flavour symmetry and in the assumption that the $\Delta B=1$ vector and axial charges at $p_{z}=\infty$ are renormalized by the factor 0.8 , derived by the semileptonic decay of $D$ mesons. It is therefore interesting 
to give the predictions within the theoretical framework assumed here. We can give definite predictions for the $C P$ violating asymmetries only for $|C|=1$ final states with the annihilation contributions taken from the data on Cabibbo favoured decays.

The mixing in the ${\overline{B_{d}}}^{0}-B_{d}^{0}$, which depends on the parameter $z_{d}=\Delta M_{d} / \Gamma_{d}=0.72 \pm 0.14$ [18], gives rise, in presence of $C P$ violation, to non-vanishing values for the asymmetries:

$$
C_{f}^{d}=\frac{\int_{0}^{\infty} d t\left\{\Gamma\left[B_{d}^{0}(t) \rightarrow f\right]-\Gamma\left[{\overline{B_{d}}}^{0}(t) \rightarrow \bar{f}\right]\right\}}{\int_{0}^{\infty} d t\left\{\Gamma\left[B_{d}^{0}(t) \rightarrow f\right]+\Gamma\left[{\overline{B_{d}}}^{0}(t) \rightarrow \bar{f}\right]\right\}}
$$

where $\Gamma\left[B_{d}^{0}(t) \rightarrow f\right]$ is the rate for producing at the instant $t$ the final state $f$ from a state, which at $t=0$ is at $B_{d}^{0}$ and $\bar{f}=(C P) f$.

One has [19] :

$$
C_{f}^{d}=\frac{2 z_{d}}{2+z_{d}^{2}+z_{d}^{2}\left|x_{f}^{d}\right|^{2}} \operatorname{Im}\left[\frac{V_{b t} V_{d t}^{*}}{V_{b t}^{*} V_{d t}} x_{f}^{d}\right]
$$

with:

$$
x_{f}^{d}=\frac{\mathcal{A}\left(B_{d}^{0} \rightarrow \bar{f}\right)}{\mathcal{A}\left({\overline{B^{0}}}_{d} \rightarrow \bar{f}\right)}=\frac{\mathcal{A}^{*}\left({\overline{B^{0}}}_{d} \rightarrow f\right)}{\mathcal{A}\left({\overline{B^{0}}}_{d} \rightarrow \bar{f}\right)}
$$

For the asymmetries associated to $B_{s}^{0}$ one can simply substitute $d \rightarrow s$ in (24) and (25).

The $x_{f}^{q}$ defined in (25) $(q=d, s)$ is given by the product of two terms:

$$
x_{f}^{q}=S_{f}^{q} R_{f}^{q}
$$

where $S_{f}^{q}$ is a function of the only CKM matrix elements and $R_{f}^{q}$ depends on the explicit computation of the matrix elements of the weak effective hamiltonian. By substituting (26) in (24) one obtains, neglecting the final state interaction:

$$
C_{f}^{q}=\frac{2 z_{q} R_{f}^{q}}{2+z_{q}^{2}+z_{q}^{2}\left(R_{f}^{q}\right)^{2}\left|S_{f}^{q}\right|^{2}} \operatorname{Im}\left[\frac{V_{b t} V_{q t}^{*}}{V_{b t}^{*} V_{q t}} S_{f}^{q}\right] .
$$

For all the final states with $|C|=1 S_{\frac{q}{f}}^{q}=1 / S_{f}^{q}$ and for all $f$ with $C=1$ we have

$$
\left|S_{f}^{s}\right|^{2}=s_{1}^{4}\left|S_{f}^{d}\right|^{2} \cong \frac{s_{2}^{2}+s_{3}^{2}+2 s_{2} s_{3} c_{\delta}}{s_{3}^{2}}
$$

In the Table 4 and 5 we report the expressions of $R_{f}^{q}$ found within the previous [6] and present approach, and the approximate expressions of $\operatorname{Im}\left[\frac{V_{b t} V_{q t}^{*}}{V_{b t}^{*} V_{q t}} S_{f}^{q}\right]$ for the decay channels with $|C|=1$ and with $\pi, \rho, K$ or $K^{*}$ in the final state. As in [8] we define

$$
w_{\pi}^{(*) 2}=\frac{m_{\pi}^{2}-\left(m_{B}-m_{D^{(*)}}\right)^{2}}{m_{B} m_{D^{(*)}}} .
$$

In Table 6 we report the values of $\xi\left(w^{2}\right)$ which appear in $R_{f}^{q}$.

As one can see in Table 4 for the decays with neutral particles in the final state, the central values of the

\footnotetext{
${ }^{5}$ We follow the Kobayashi-Maskawa parameterization reported also in [10]
} 
annihilation contributions are of the same order of magnitude than the direct ones, which are proportional to $k_{2} \cong-0.25$ according to equations (3), and their relative sign changes, when we change the initial (or the final state) into its $C P$ conjugate. In particular for $D^{0}\left(D^{* 0}\right) \pi^{0}$ and $D^{0} \rho^{0}$ final states the Cabibbo allowed amplitudes are expected to be small and with uncertain sign in such a way to allow for values of $\left|x_{f}^{d}\right|=\left|R_{f}^{d} S_{f}^{d}\right| \cong 1$ and consequently for large asymmetries of either sign.

Instead for the final states with charged particles we are able to predict the sign and the order of magnitude of the corresponding $R_{f}^{q}$. The predictions for $C_{f}^{d}$ and $C_{f}^{s}$ with the values of the KobayashiMaskawa parameters

$$
s_{2}=0.050 \quad s_{3}=0.023 \quad s_{\delta}=0.894 \quad c_{\delta}=-0.448
$$

are reported in Tables 7 and 8 respectively. In correspondence of $\xi^{p a r}\left(w^{2}\right)$ the branching ratios and the minimum number of $b \bar{b}$-couples needed for a $3 \sigma$-evidence of the asymmetries are also computed.

\section{Conclusion}

The measured branching ratios for two body nonleptonic decays of B-particles are well described, in absence of final state interaction, by assuming factorization, total colour screening and with the matrix elements of the weak currents given by Isgur and Wise theory and $S U(2)_{H F}$. We allow for $S U(3)$ invariant contributions of the annihilation terms to be fitted from data, which come out larger, as in charm decay [4], than the values predicted by assuming the dominance of the lowest $0^{ \pm}$octets [17]. Between the parameterization considered for the universal function $\xi\left(w^{2}\right)$ the parabolic one is slightly favoured, expecially for the spectrum in the $e \bar{\nu}$ invariant mass for the decay $\overline{B^{0}} \rightarrow D^{+*} e^{-} \bar{\nu}_{e}$. This parameterization is also the one giving $\left|V_{b c}\right|$ the value 0.044 in best agreement with present determinations.

Indeed, also with a $\xi\left(w^{2}\right)$ with the pole corresponding to the $b \bar{c}$ resonances and $\xi(0) \neq 1$ one gets a good fit, just showing to us that the large errors in the data cannot at the moment allow for drastic conclusions. Instead within the previous approach based on $S U(4)_{(u, d, s, c)}$ one predicts for the rate $B \rightarrow J / \Psi K$ larger values than experiment, which casts doubts on its validity despite its capability in describing the rates into final states with $|C|=1$. With the parameters fixed from the measured decays we are able to predict the $C P$ violating asymmetries in the decays of neutral $B$-particles into $|C|=1$ final states in absence of final state interaction. Large asymmetries are expected for the final states $D^{(*) 0} \pi^{0}$ and $D^{0} \rho^{0}$, but the present uncertainties do not allow to predict even the sign.

We have better confidence in our predictions on sign and order of magnitude of the asymmetries for the decays with charged particles in the final state. 


\section{References}

[1 ]N. Isgur and M.B. Wise Phys. Lett. B232(1989)113;

N. Isgur and M.B. Wise Phys. Lett. B237(1990)527;

[2 ]M. Neubert, Phys. Lett. B264(1991)455;

[3 ] Argus Collaboration data presented by H. Scroder to Desy Workshop (Hamburg 1992);

[4 ]F. Buccella,M.Lusignoli, G. Miele and A. Pugliese, Zeit. Phys. C 55(1992)243;

[5 ]J.C. Anjos et al. (E691 Collaboration), Phys. Rev. Lett. 62(1989)1587 and 65(1990)2630;

[6 ]N. Isgur and M.B. Wise, Phys. Rev. D42(1990)2388;

[7 ]M. Tanimoto, Phys. Rev. D44(1991)1449;

[8 ]F. Buccella,F. Lombardi and P.Santorelli, Il Nuovo Cimento A, 105(1992)993,

G.Burdman, Phys. Lett. B284(1992)133;

[9 ]J. Rosner, Phys. Rev. D42(1990),3732;

T.Mannel, W.Roberts and Z.Rizak, Phys. Lett. B259(1991),359;

[10 ]F. Buccella,G.Mangano and G. Miele, Il Nuovo Cimento A, 104(1991)1293;

F. Buccella,G.Mangano,G. Miele and P.Santorelli, Il Nuovo Cimento A 105(1992)33;

[11 ]A.F.Falk, H.Georgi, B.Grinstein and M.B.Wise, Nucl. Phys. B343(1990)1;

[12 ]G.Altarelli, G.Curci, G.Martinelli and S.Petrarca, Nucl. Phys. B187(1981)461;

[13 ]V. Lubicz, G. Martinelli and C.T. Sachrajda, Rome Preprint n. 748, July 11th, 1990;

[14 ]M. Bauer and B. Stech, Phys. Lett. B152(1985)380;

M. Bauer, B. Stech and Wirbel, Zeit. fur Phys. C34(1987)103;

[15 ]Particle Data Group, Phys. Rev. D45(1992)Part 2;

[16 ]J.G. Korner, K.Schilcher, M.Wirbel and J.L.Wu, Zeit. Phys. C 48(1990)663;

[17 ]D.Fakirov and B.Stech, Nucl. Phys. B133(1978)315;

[18 ]H.Albrecht et al.,Phys. Lett.B192(1987)245;

[19 ]A.B.Carter and A.I.Sanda,Phys.Rev.Lett.45(1980)952, Phys. Rev.D23(1981)1567;

A.I.Bigi and A.I.Sanda,Nucl.Phys.B193(1981)85. 
Table 1

\begin{tabular}{|c|c|c|c|}
\hline Form & Residua & $\begin{array}{c}M_{c \bar{q}}\left(J^{P}\right) \\
\{\bar{q}=\bar{s}\} \\
G e V\end{array}$ & $\begin{array}{c}M_{c \bar{q}}\left(J^{P}\right) \\
\{\bar{q}=\bar{u}, \bar{d}\} \\
G e V\end{array}$ \\
\hline Factors & $0.79 \pm 0.08$ & $2.60\left(0^{+}\right)$ & $2.47\left(0^{+}\right)$ \\
$f_{0}^{(c q)}\left(q^{2}\right)$ & $2.11\left(1^{-}\right)$ & $2.01\left(1^{-}\right)$ \\
$f_{1}^{(c q)}\left(q^{2}\right)$ & $0.79 \pm 0.08$ & $1.97\left(0^{-}\right)$ & $1.87\left(0^{-}\right)$ \\
$A_{0}^{(c q)}\left(q^{2}\right)$ & $0.71 \pm 0.16$ & $2.53\left(1^{+}\right)$ & $2.42\left(1^{+}\right)$ \\
$A_{1}^{(c q)}\left(q^{2}\right)$ & $0.46 \pm 0.07$ & $2.53\left(1^{+}\right)$ & $2.42\left(1^{+}\right)$ \\
$A_{2}^{(c q)}\left(q^{2}\right)$ & $0.00 \pm 0.22$ & $2.11\left(1^{-}\right)$ & $2.01\left(1^{-}\right)$ \\
$V^{(c q)}\left(q^{2}\right)$ & $0.90 \pm 0.32$ & 2.06 \\
\hline
\end{tabular}


Table 2

\begin{tabular}{|c|c|c|c|c|}
\hline & $\xi^{\text {par }}\left(w^{2}\right)$ & $\xi^{\exp }\left(w^{2}\right)$ & $\xi^{\text {pole }}\left(w^{2}\right)$ & $\xi_{\text {pole }}^{\text {phys. }}\left(w^{2}\right)$ \\
\hline & $\left\{\begin{array}{l}b=1.06_{-0.23}^{+0.20} \\
c=0.62_{-0.20}^{+0.17}\end{array}\right.$ & $\beta=0.48_{-0.14}^{+0.15}$ & $w_{0}=1.20_{-0.17}^{+0.27}$ & $\xi(0)=0.83_{-0.09}^{+0.14}$ \\
\hline$\left|V_{b c}\right|$ & $0.044 \pm 0.004$ & $0.037 \pm 0.003$ & $0.039 \pm 0.004$ & $0.040 \pm 0.005$ \\
\hline$W_{P P}$ & $-0.078_{-0.021}^{+0.019}$ & $-0.028_{-0.025}^{+0.022}$ & $-0.032_{-0.024}^{+0.021}$ & $-0.051_{-0.023}^{+0.021}$ \\
\hline$W_{D V}$ & $+0.115_{-0.152}^{+0.105}$ & $+0.047_{-0.181}^{+0.125}$ & $+0.051_{-0.172}^{+0.119}$ & $+0.085_{-0.168}^{+0.116}$ \\
\hline$W_{D^{*} P}$ & $-0.054_{-0.025}^{+0.022}$ & $-0.042_{-0.029}^{+0.026}$ & $-0.042_{-0.028}^{+0.025}$ & $-0.058_{-0.027}^{+0.024}$ \\
\hline $\begin{array}{c}\chi^{2} \\
\left(\chi^{2} / N d f\right)\end{array}$ & $\begin{array}{l}13.35 \\
(0.89)\end{array}$ & $\begin{array}{l}18.87 \\
(1.18)\end{array}$ & $\begin{array}{l}17.83 \\
(1.11)\end{array}$ & $\begin{array}{l}18.71 \\
(1.17)\end{array}$ \\
\hline$\chi_{\text {spectrum }}^{2}$ & 6.79 & 8.01 & 7.54 & 11.05 \\
\hline
\end{tabular}

The order of magnitude of the analogous of $W$ 's estimated in [17] and [10] are $W_{P P} \cong-0.002, W_{D V}=W_{D^{*} P} \cong-0.010$ 
Table 3

\begin{tabular}{|c|c|c|c|c|c|}
\hline \multirow[b]{2}{*}{ Channels } & \multirow[t]{2}{*}{ Exp. rates [15] } & \multicolumn{4}{|c|}{ Theoretical rates } \\
\hline & & $\begin{array}{c}\xi^{\text {par }}\left(w^{2}\right) \\
\left(10^{-12} \mathrm{MeV}\right)\end{array}$ & $\begin{array}{c}\xi^{e x p}\left(w^{2}\right) \\
\left(10^{-12} \mathrm{MeV}\right)\end{array}$ & $\begin{array}{c}\xi^{\text {pole }}\left(w^{2}\right) \\
\left(10^{-12} \mathrm{MeV}\right)\end{array}$ & $\begin{array}{c}\xi_{\text {pole }}^{\text {phys. }}\left(w^{2}\right) \\
\left(10^{-12} \mathrm{MeV}\right)\end{array}$ \\
\hline $\begin{array}{l}\frac{B^{-}}{B_{d}^{0}} \rightarrow D^{0} D_{s}^{-} \\
\rightarrow D^{+} D_{s}^{-}\end{array}$ & $\left.\begin{array}{l}9.1 \pm 5.3 \\
3.7 \pm 2.3\end{array}\right\}$ & 4.2 & 4.7 & 4.6 & 4.9 \\
\hline$B^{-} \rightarrow D^{0} \pi^{-}$ & $1.81 \pm 0.53$ & 1.81 & 1.06 & 1.08 & 1.34 \\
\hline$\overline{B_{d}^{0}} \rightarrow D^{+} \pi^{-}$ & $1.48 \pm 0.32$ & 1.48 & 1.48 & 1.48 & 1.48 \\
\hline$\overline{B_{d}^{0}} \rightarrow D_{s}^{+} K^{-}$ & $<0.62$ & 0.27 & 0.03 & 0.04 & 0.09 \\
\hline $\begin{array}{l}\frac{B^{-}}{B_{d}^{0}} \rightarrow J / \Psi K^{-} \\
\rightarrow \Psi \overline{K^{0}}\end{array}$ & $\left.\begin{array}{l}0.37 \pm 0.10 \\
0.30 \pm 0.14\end{array}\right\}$ & 0.38 & 0.27 & 0.30 & 0.31 \\
\hline$\overline{B_{d}^{0}} \rightarrow D^{+*} D_{s}^{-}$ & $7.4 \pm 5.1$ & 2.83 & 3.29 & 3.24 & 3.23 \\
\hline$B^{-} \rightarrow D^{0} \rho^{-}$ & $6.2 \pm 2.9$ & 4.83 & 3.36 & 3.40 & 4.07 \\
\hline$\overline{B_{d}^{0}} \rightarrow D^{+} \rho^{-}$ & $4.2 \pm 2.8$ & 4.17 & 4.17 & 4.17 & 4.17 \\
\hline$\overline{B_{d}^{0}} \rightarrow D^{0} \rho^{0}$ & $<0.28$ & 0.010 & 0.021 & 0.019 & 0. \\
\hline$B^{-} \rightarrow D^{0 *} \pi^{-}$ & $2.5 \pm 0.7$ & 1.31 & 1.20 & 1.18 & 1.37 \\
\hline$\overline{B_{d}^{0}} \rightarrow D^{+*} \pi^{-}$ & $1.48 \pm 0.32$ & 1.48 & 1.48 & 1.48 & 1.48 \\
\hline
\end{tabular}


Table 4

\begin{tabular}{|c|c|c|c|}
\hline$f$ & $\begin{array}{c}\quad R_{f}^{d} \\
\text { cfr. }[10]\end{array}$ & $R_{f}^{d}$ & $\operatorname{Im}\left[\frac{V_{b t} V_{d t}^{*}}{V_{b t}^{*} V_{d t}} S_{f}^{d}\right] \cong$ \\
\hline$D^{+} \pi^{-}$ & 0.49 & $\left(\frac{\left.0.43 \xi\left(w_{\pi}^{2}\right)+W_{P P}\right)}{0.27-W_{P P}}\right)$ & $-\frac{s_{\delta}\left(s_{2}+2 s_{3} c_{\delta}\right)}{s_{1}^{2} s_{3}}$ \\
\hline$D^{-} \pi^{+}$ & 2.06 & $\left(\frac{0.27-W_{P P}}{0.43 \xi\left(w_{\pi}^{2}\right)+W_{P P}}\right)$ & $-\frac{s_{1}^{2} s_{3} s_{\delta}\left(s_{2}+2 s_{3} c_{\delta}\right)}{s_{3}^{2}+s_{2}^{2}+2 s_{2} s_{3} c_{\delta}}$ \\
\hline$D^{0} \pi^{0}$ & 1.04 & $\left(\frac{0.06+W_{P P}}{0.06-W_{P P}}\right)$ & $-\frac{s_{\delta}\left(s_{2}+2 s_{3} c_{\delta}\right)}{s_{1}^{2} s_{3}}$ \\
\hline $\bar{D}^{0} \pi^{0}$ & 0.96 & $\left(\frac{0.06-W_{P P}}{0.06+W_{P P}}\right)$ & $-\frac{s_{1}^{2} s_{3} s_{\delta}\left(s_{2}+2 s_{3} c_{\delta}\right)}{s_{3}^{2}+s_{2}^{2}+2 s_{2} s_{3} c_{\delta}}$ \\
\hline$D_{s}^{+} K^{-}$ & -1 & -1 & $\frac{s_{2} s_{\delta}}{s_{3}}$ \\
\hline$D_{s}^{-} K^{+}$ & -1 & -1 & $\frac{s_{2} s_{3} s_{\delta}}{s_{3}^{2}+s_{2}^{2}+2 s_{2} s_{3} c_{\delta}}$ \\
\hline$D^{0 *} \pi^{0}$ & 1.17 & $\left(\frac{0.07+W_{D^{*} P}}{0.07-W_{D^{*} P}}\right)$ & $\frac{s_{\delta}\left(s_{2}+2 s_{3} c_{\delta}\right)}{s_{1}^{2} s_{3}}$ \\
\hline $\bar{D}^{0 *} \pi^{0}$ & 0.85 & $\left(\frac{0.07-W_{D^{* P}}}{0.07+W_{D^{*} P}}\right)$ & $\frac{s_{1}^{2} s_{3} s_{\delta}\left(s_{2}+2 s_{3} c_{\delta}\right)}{s_{3}^{2}+s_{2}^{2}+2 s_{2} s_{3} c_{\delta}}$ \\
\hline$D^{0} \rho^{0}$ & 1.18 & $\left(\frac{0.09-W_{D V}}{0.09+W_{D V}}\right)$ & $\frac{s_{\delta}\left(s_{2}+2 s_{3} c_{\delta}\right)}{s_{1}^{2} s_{3}}$ \\
\hline $\bar{D}^{0} \rho^{0}$ & 0.85 & $\left(\frac{0.09+W_{D V}}{0.09-W_{D V}}\right)$ & $\frac{s_{1}^{2} s_{3} s_{\delta}\left(s_{2}+2 s_{3} c_{\delta}\right)}{s_{3}^{2}+s_{2}^{2}+2 s_{2} s_{3} c_{\delta}}$ \\
\hline$D^{+} \rho^{-}$ & 0.97 & $\left(\frac{0.81 \xi\left(w_{\rho}^{2}\right)-W_{D V}}{0.40+W_{D V}}\right)$ & $\frac{s_{\delta}\left(s_{2}+2 s_{3} c_{\delta}\right)}{s_{1}^{2} s_{3}}$ \\
\hline$D^{-} \rho^{+}$ & 1.03 & $\left(\frac{0.40+W_{D V}}{0.81 \xi\left(w_{\rho}^{2}\right)-W_{D V}}\right)$ & $\frac{s_{1}^{2} s_{3} s_{\delta}\left(s_{2}+2 s_{3} c_{\delta}\right)}{s_{3}^{2}+s_{2}^{2}+2 s_{2} s_{3} c_{\delta}}$ \\
\hline$D^{+*} \pi^{-}$ & 0.51 & $\left(\frac{0.48 \xi\left(w_{\pi}^{* 2}\right)+W_{D^{*} P}}{0.30-W_{D^{*} P}}\right)$ & $\frac{s_{\delta}\left(s_{2}+2 s_{3} c_{\delta}\right)}{s_{1}^{2} s_{3}}$ \\
\hline$D^{-*} \pi^{+}$ & 1.95 & $\left(\frac{0.30-W_{D^{*} P}}{0.48 \xi\left(w_{\pi}^{* 2}\right)+W_{D^{*} P}}\right)$ & $\frac{s_{1}^{2} s_{3} s_{\delta}\left(s_{2}+2 s_{3} c_{\delta}\right)}{s_{3}^{2}+s_{2}^{2}+2 s_{2} s_{3} c_{\delta}}$ \\
\hline
\end{tabular}


Table 5

\begin{tabular}{|c|c|c|c|}
\hline$f$ & $\begin{array}{c}R_{f}^{s} \\
c f r .[10]\end{array}$ & $R_{f}^{s}$ & $\operatorname{Im}\left[\frac{V_{b t} V_{s t}^{*}}{V_{b t}^{*} V_{s t}} S_{f}^{s}\right] \cong$ \\
$D_{s}^{+} K^{-}$ & 1.59 & $\left(\frac{0.54 \xi\left(w_{K}^{2}\right)+W_{P P}}{0.26-W_{P P}}\right)$ & $\frac{s_{2} s_{\delta}}{s_{3}}$ \\
$D_{s}^{-} K^{+}$ & 0.63 & $\left(\frac{0.26-W_{P P}}{0.54 \xi\left(w_{K}^{2}\right)+W_{P P}}\right)$ & $\frac{s_{2} s_{3} s_{\delta}}{s_{2}^{2}+s_{3}^{2}+2 s_{2} s_{3} c_{\delta}}$ \\
$D_{s}^{+*} K^{-}$ & 0.66 & $\left(\frac{0.61 \xi\left(w_{K}^{2}\right)+W_{D^{*} P}}{0.29-W_{D^{*} P}}\right)$ & $-\frac{s_{2} s_{\delta}}{s_{3}}$ \\
$D_{s}^{-*} K^{+}$ & 1.52 & $\left(\frac{0.54 \xi\left(w_{K}^{2}\right)+W_{P P}}{0.26-W_{P P}}\right)$ & $-\frac{s_{2} s_{3} s_{\delta}}{s_{2}^{2}+s_{3}^{2}+2 s_{2} s_{3} c_{\delta}}$ \\
$D_{s}^{+} K^{-*}$ & 0.73 & $\left(\frac{0.39 \xi\left(w_{K^{*}}^{2}\right)-W_{D V}}{0.30+W_{D V}}\right)$ & $-\frac{s_{2} s_{\delta}}{s_{3}}$ \\
$D_{s}^{-} K^{+*}$ & 1.38 & $\left(\frac{0.30+W_{D V}}{0.39 \xi\left(w_{K^{*}}^{2}\right)-W_{D V}}\right)$ & $-\frac{s_{2} s_{3} s_{\delta}}{s_{2}^{2}+s_{3}^{2}+2 s_{2} s_{3} c_{\delta}}$ \\
\hline
\end{tabular}

Table 6

\begin{tabular}{|c|c|c|c|c|}
\hline & $\xi^{\text {par }}\left(w^{2}\right)$ & $\xi^{\text {exp }}\left(w^{2}\right)$ & $\xi^{\text {pole }}\left(w^{2}\right)$ & $\xi_{\text {pole }}^{\text {phys. }}\left(w^{2}\right)$ \\
\hline$w_{\pi}^{2}$ & 0.611 & 0.569 & 0.550 & 0.588 \\
\hline$w_{\rho}^{2}$ & 0.590 & 0.585 & 0.563 & 0.596 \\
\hline$w_{K}^{2}$ & 0.577 & 0.597 & 0.572 & 0.603 \\
\hline$w_{K^{*}}^{2}$ & 0.565 & 0.612 & 0.584 & 0.611 \\
\hline$w_{\pi}^{* 2}$ & 0.561 & 0.617 & 0.589 & 0.614 \\
\hline
\end{tabular}


Table 7

\begin{tabular}{|c|c|c|c|c|c|}
\hline $\begin{array}{c}C_{f}^{d} \\
f\end{array}$ & $\xi^{p a r}\left(w^{2}\right)$ & $\xi^{e x p}\left(w^{2}\right)$ & $\xi^{\text {pole }}\left(w^{2}\right)$ & $\begin{array}{c}\operatorname{Br}\left(B_{d}^{0}, f_{i}\right) \\
\%\end{array}$ & $\sigma\left(B_{d}^{0} B^{-}\right) \epsilon N_{b \bar{b}}$ \\
\hline$D^{+} \rho^{-}$ & 0.059 & 0.043 & 0.046 & $0.1210^{-2}$ & $8.010^{5}$ \\
\hline$D^{+} \pi^{-}$ & -0.079 & -0.058 & -0.062 & $0.7610^{-3}$ & $1.210^{6}$ \\
\hline$D^{+*} \pi^{-}$ & 0.068 & 0.056 & 0.059 & $0.5610^{-3}$ & $1.710^{6}$ \\
\hline$D^{-} \rho^{+}$ & 0.012 & 0.009 & 0.010 & 0.96 & $3.810^{6}$ \\
\hline$D^{-} \pi^{+}$ & -0.016 & -0.012 & -0.013 & 0.34 & $5.910^{6}$ \\
\hline$D^{-*} \pi^{+}$ & 0.014 & 0.012 & 0.012 & 0.340 & $8.110^{6}$ \\
\hline$D_{s}^{+} K^{-}$ & 0.042 & 0.042 & 0.042 & $0.3710^{-4}$ & $2.510^{7}$ \\
\hline$D_{s}^{-} K^{+}$ & 0.009 & 0.009 & 0.009 & $0.6110^{-1}$ & $1.210^{8}$ \\
\hline$D^{0} \rho^{0}$ & -0.285 & 0.130 & 0.146 & $0.9410^{-4}$ & $1.210^{7}$ \\
\hline$D^{0} \pi^{0}$ & 0.284 & -0.111 & -0.131 & $0.6110^{-4}$ & $1.810^{7}$ \\
\hline$D^{0 *} \pi^{0}$ & 0.311 & 0.174 & 0.174 & $0.3310^{-4}$ & $3.510^{7}$ \\
\hline $\bar{D}^{0} \rho^{0}$ & -0.070 & 0.028 & 0.031 & $0.2310^{-2}$ & $4.810^{7}$ \\
\hline $\bar{D}^{0} \pi^{0}$ & 0.069 & -0.023 & -0.028 & $0.1510^{-2}$ & $7.410^{7}$ \\
\hline $\bar{D}^{0 *} \pi^{0}$ & 0.080 & 0.038 & 0.038 & $0.6210^{-3}$ & $1.410^{8}$ \\
\hline
\end{tabular}


Table 8

\begin{tabular}{|c|c|c|c|c|c|}
\hline$C_{f}^{s}$ & $\xi^{\text {par }}\left(w^{2}\right)$ & $\xi^{\exp }\left(w^{2}\right)$ & $\xi^{\text {pole }}\left(w^{2}\right)$ & $\operatorname{Br}\left(B_{s}^{0}, f_{i}\right)$ & $\sigma\left(B_{s}^{0} B^{-}\right) \epsilon N_{b \bar{b}}$ \\
$f$ & & & & $\%$ & \\
\hline$D_{s}^{+} K^{-}$ & 0.079 & 0.066 & 0.069 & $0.1510^{-1}$ & $3.310^{6}$ \\
\hline$D_{s}^{-} K^{+}$ & 0.079 & 0.065 & 0.068 & $0.2910^{-1}$ & $3.310^{6}$ \\
\hline$D_{s}^{-} K^{+*}$ & -0.082 & -0.067 & -0.070 & $0.2010^{-1}$ & $3.810^{6}$ \\
\hline$D_{s}^{+} K^{-*}$ & -0.082 & -0.067 & -0.070 & $0.1510^{-1}$ & $3.810^{6}$ \\
\hline$D_{s}^{+*} K^{-}$ & -0.074 & -0.064 & -0.067 & $0.1110^{-1}$ & $4.110^{6}$ \\
\hline$D_{s}^{-*} K^{+}$ & -0.073 & -0.064 & -0.066 & $0.3010^{-1}$ & $4.110^{6}$ \\
\hline
\end{tabular}

To compute these asymmetries we assume $\tau_{B_{d}}=\tau_{B_{s}}$ and $z_{s}=z_{d} \frac{\left|V_{s t}\right|^{2}}{\left|V_{d t}\right|^{2}}$. 